\title{
¿Qué aportan las Tecnologías de la Información y Comunicación en la enseñanza de las ciencias?
}

Héctor Medina Cruz, Agustín Lagunes Domínguez y María Teresa Guerra Ramos

\begin{abstract}
Resumen
En este trabajo se discute sobre la creciente integración de las Tecnologías de la Información y Comunicación (TIC) en el ámbito educativo, las grandes expectativas que han generado desde su llegada, así como sus aportaciones y potencial para una formación científica de niños y adolescentes orientada a la toma de decisiones, al pensamiento crítico y a la actuación responsable. Se comentan las diferentes iniciativas en México para integrar las TIC en escuelas de educación básica y se recuperan algunos datos de la infraestructura necesaria tanto en primarias como en secundarias. Posteriormente, nos concentramos en analizar las posibilidades que las tıc ofrecen en la educación; específicamente, a la educación en ciencias, asumiendo una postura socioconstructivista.
\end{abstract}

Palabras clave: Tecnologías de la Información y Comunicación, educación básica, ciencias, Tıc.

\section{How do Information and Communication TeChNologies contribute to SCIENCE EDUCATION?}

\begin{abstract}
This paper presents a discussion about the growing integration of Information and Communication Technologies (IcT) in education, the great expectations that these have generated since their arrival, as well as their contributions and potential for scientific education of children and adolescents oriented to decision making, critical thinking and responsible action. To do this, we discuss the different initiatives to integrate Ict in basic education schools in Mexico, and we recover some data on Ict infrastructure in primary and secondary schools. We also focus on analyzing the potential of Ict for education; particularly, to science education, assuming a constructivist social position.
\end{abstract}

Keywords: Information and Communication Technologies, basic education, sciences, ICT

Dol: http://doi.org/10.22201/codeic.16076079e.2020.v21n3.a9 


\section{Héctor Medina Cruz}

Benemérita Universidad Autónoma de Puebla (BUAP), Coordinador del Colegio de Ingeniería en Procesos y Gestión Industrial (IPGI). Es Ingeniero Industrial por el Instituto Tecnológico de Puebla. Maestro en Administración de la Manufactura por la Universidad de las Américas Puebla. Doctor en Sistemas y Ambientes Educativos (DSAE) por la Universidad Veracruzana. Profesor Investigador Asociado C de Tiempo Completo.

\section{Agustín Lagunes Domínguez}

aglagunes@uv.mx

Universidad Veracruzana, Coordinador del Doctorado en Sistemas y Ambientes Educativos (PNPC). Licenciado en Informática y Maestro en Ciencias en Ciencias Computacionales por el Instituto Tecnológico de Orizaba, Doctor en Sistemas y Ambientes Educativos (DSAE) con mención Cum Laude por la Universidad Autónoma de Madrid. Profesor investigador de tiempo completo de la Universidad Veracruzana.

\section{María Teresa Guerra Ramos}

Centro de Investigación y de Estudios Avanzados, Unidad Monterrey, profesora-investigadora de tiempo completo. Es licenciada en Psicología egresada de la Facultad de Psicología de la unam. Maestra en Educación y Doctora en Educación en Ciencias por el centro de Estudios en Educación Científica y Matemática de la Universidad de Leeds, Inglaterra. Fundadora de la Maestría en Educación en Biología para la Formación Ciudadana del Cinvestav Monterrey. 


\section{Introducción}

México es un país con muchos desafíos pendientes en materia educativa, ante un marco de elevados índices de pobreza y una creciente desigualdad social. Las prioridades en educación incluyen cuestiones como la deserción escolar, el analfabetismo en adultos, la calidad educativa, los programas de formación del profesorado y la necesidad de introducir las Tecnologías de la Información y Comunicación (TIC).

La incorporación de las tic en el nivel educativo básico en México no resulta un tema nuevo. Desde los inicios de la educación básica en 1927, con la conformación de la Secretaría de Educación Pública (SEP) -que tuvo como objetivo promover la alfabetización y la extensión de la enseñanza en zonas rurales- se han integrado diversos dispositivos tecnológicos, acordes a cada época, con fines pedagógicos y educativos. Habría que mencionar, por ejemplo, la creación de la Dirección General de Educación Audiovisual (DGEAv), en el año 1965, la cual buscó nuevas alternativas de educación con el fin de abatir el rezago educativo y la alfabetización, principalmente en áreas rurales a través de medios de comunicación como la radio y la televisión. Calixto y Albarrón (2008) relatan cómo en esa época la televisión se utilizó como recurso didáctico cuyo fin era cubrir el aprendizaje de un determinado nivel escolar, mediante la transmisión de programas educativos.

Sin embargo, como menciona Díaz (2014), en los años recientes la aparición de internet, celulares y televisores inteligentes, recursos digitales y su gran disponibilidad entre las nuevas generaciones, han originado nuevos procesos masivos de socialización, marcando un empuje de las TIC en los procesos educativos, lo que representa una posibilidad para el enriquecimiento de la educación en ciencias.

Este artículo tiene como propósito revisar, primero, el panorama general del uso de las Tic en la educación básica y, posteriormente, mencionar algunas características de las TIc, que podrían mejorar el aprendizaje y la enseñanza de las ciencias en el marco y perspectiva socioconstructivista.

\section{Las TIC y la educación básica en México}

Gran parte de los países en América Latina, incluyendo México, cuenta con programas o iniciativas para la integración de TIC en sus sistemas educativos (Lugo, Kelly y Schurmann, 2012). Estas políticas se basan en una fuerte reflexión y análisis social, que en la mayoría de los casos sobrepasa los propósitos pedagógicos, es decir, primero toman en cuenta el objetivo de democratizar el acceso a las Tıc y, en segunda instancia, se enfocan en las transformaciones pedagógicas. 
Dichas políticas se reflejan en México desde principios de los años noventa, entre los que destaca el proyecto Red Escolar, auspiciado por la SEP con el apoyo del Instituto Latinoamericano de la Comunicación Educativa (ILCE). En este programa, comenta Díaz (2014), las metas residían en la apertura del acceso a la conectividad y en la provisión de equipo de cómputo a las escuelas.

Como otra tendencia importante, se priorizó la creación de portales educativos y contenidos digitales dirigidos a los docentes, estudiantes y, en ocasiones, a los padres u otros agentes educativos (García y Rincón, 2006). De esta forma, a inicios del año 2000, la SEP y el ILCE establecieron el programa sepiensa, portal educativo de educación básica y media para México.

Durante los años 2000 a 2012, Díaz (2014) enfatiza la importancia del proyecto Enciclomedia, que dotaba a escuelas primarias públicas, de distintos estados, con aulas equipadas con computadora, proyector, pizarrón electrónico, fuente de poder, mesa de cómputo e impresora. Los docentes a cargo de los grupos daban clases utilizando dichos recursos, además, los contenidos digitales estaban precargados en el disco duro de la computadora, por lo que no había necesidad de conectarse a la red si esto no era posible.

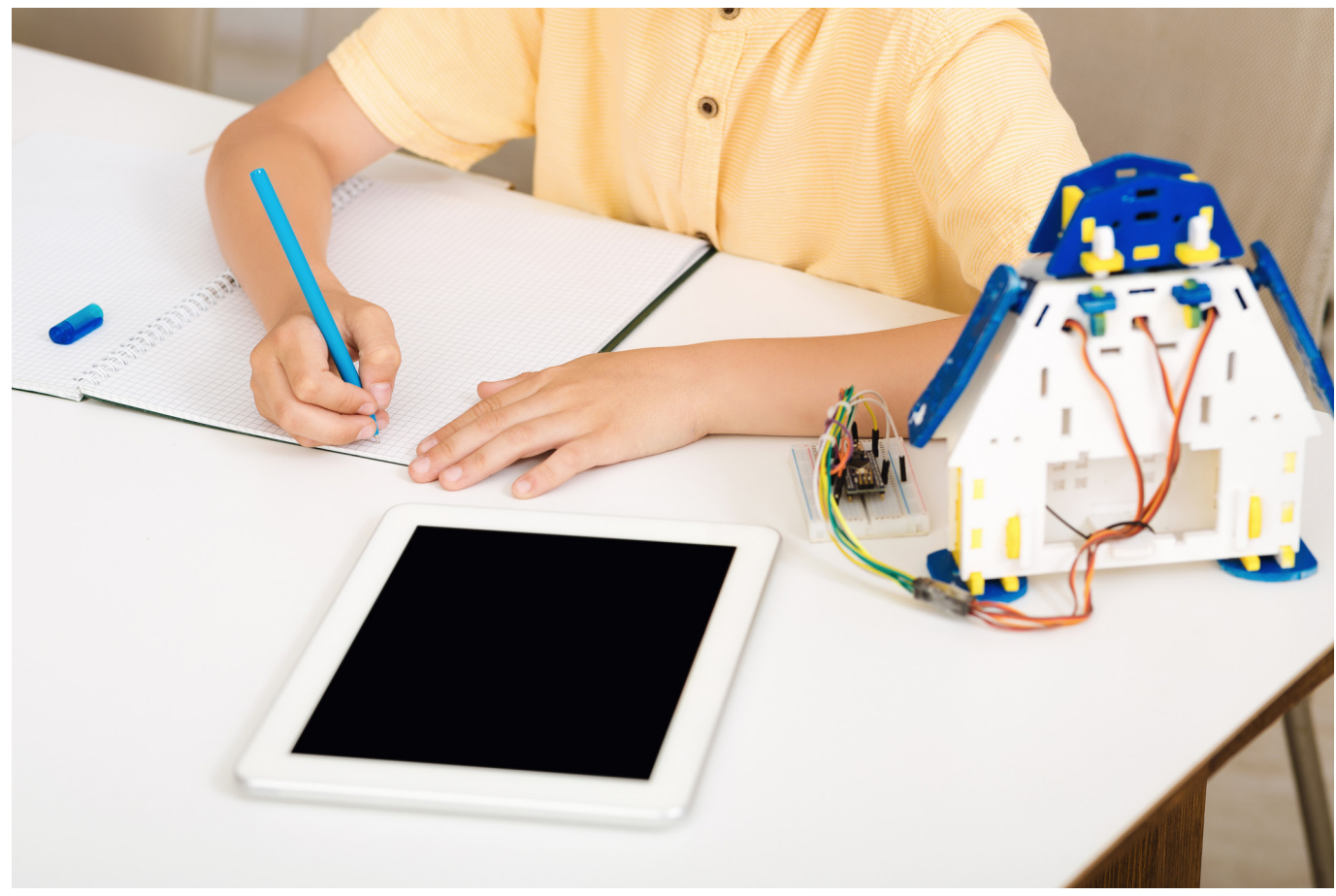

En 2008, el gobierno federal y la SEP implementaron el programa Habilidades Digitales para Todos (HDT) en telesecundarias, considerado por cierto sector como el sustituto de Enciclomedia. Éste otorgaba al docente, de quinto y sexto de primaria, computadora, proyector y pizarrón electrónico, parecido a lo que hacía Enciclomedia, así como conectividad y acceso al portal local o de aula denominado Explora. 
"¿Qué aportan las Tecnologías de la Información y Comunicación en la enseñanza de las ciencias?" Héctor Medina Cruz, Agustín Lagunes Domínguez y María Teresa Guerra Ramos

Vol. 21, Núm. 3, mayo-junio 2020 Revista Digital Universitaria

Posteriormente, a partir del año 2013, como proyecto sello de la política en materia educativa, se creó una estrategia llamada Mi Compu.MX cuyo objetivo era proporcionar computadoras portátiles a los estudiantes de escuelas públicas mexicanas de nivel básico, a las cuales también se les garantizaría el acceso a internet. Otra variante de este programa dio tablets a estudiantes de educación primaria que cursaban el quinto grado. La iniciativa fue parte de los programas del gobierno federal, específicamente de la estrategia digital nacional que buscaba promover la adopción y desarrollo de las tic durante el período 2014-2018 (SEP, 2013).

Como se ha mencionado, junto con la implementación de diversos programas y modelos de inclusión de TIc, el gobierno mexicano ha realizado esfuerzos por dotar de infraestructura tecnológica a las escuelas de educación básica. Entre esas iniciativas se encuentran: Red escolar (1998 a 2004), Enciclomedia (2004-2011), Habilidades Digitales para Todos (2009-2012), Mi Compu.MX (2013-2014), Programa Piloto de Inclusión Digital (2013-2015), Programa @prende.mx (2014-2016)y Programa @prende 2.0 (2016-2017), entre otras (sEP, 2016). Sin embargo, diversos indicadores muestran que las acciones han sido insuficientes. Por ejemplo, el índice del Instituto Nacional de Evaluación Educativa (INEE) del año 2018, denominado Porcentaje de escuelas primarias y secundaria reportadas oficialmente con una computadora para uso educativo, sugiere que en el ciclo escolar anterior a dicho índice no hay un crecimiento evidente de tales herramientas tecnológicas en los centros escolares y que existe un porcentaje menor en la educación primaria (ver tabla 1)

Tabla 1. Porcentajes de primarias y secundarias con al menos una computadora para uso educativo en los ciclos escolares 2011 a 2018. Fuente: elaboración propia con datos del INEE (2019).

\begin{tabular}{|l|c|c|}
\hline Ciclo escolar & $\begin{array}{c}\text { \% de primarias con al } \\
\text { menos una computadora } \\
\text { para uso educativo }\end{array}$ & $\begin{array}{c}\text { \% de secundarias con al } \\
\text { menos una computadora } \\
\text { para uso educativo }\end{array}$ \\
\hline $2011-2012$ & 46.0 & 71.7 \\
\hline $2012-2013$ & 43.2 & 70.4 \\
\hline $2013-2014$ & 39.6 & 68.4 \\
\hline $2015-2016$ & 40.4 & 70.1 \\
\hline $2017-2018$ & 46.5 & 74.7 \\
\hline
\end{tabular}

Dentro del nivel primaria, hay grandes disparidades: mientras que casi la mitad de las primarias generales cuentan con computadora (48.5\%), únicamente $31.9 \%$ de las escuelas indígenas trabajan con al menos una computadora con uso educativo, y en los cursos comunitarios prácticamente la presencia de estos dispositivos digitales es nula con un 1.7\% (INEE, 2019).

A pesar de que el sistema de secundarias tiene una mayor cobertura que el de primarias, de por lo menos una computadora para uso educativo -las secundarias generales y técnicas cuentan con porcentajes de acceso a una computadora de $83.1 \%$ y $79.1 \%$, respectivamente-, éste se reduce en las telesecundarias a $68.7 \%$, y comienza a escasear en las secundarias de servicio educativo para trabajadores con 28.2\% (INEE, 2019). 
“Qué aportan las Tecnologías de la Información y Comunicación en la enseñanza de las ciencias?" Héctor Medina Cruz, Agustín Lagunes Domínguez y María Teresa Guerra Ramos

Sobre la conectividad de internet, el Censo de Escuelas, Maestros, Alumnos de educación Básica y Especial (CEMABE), con los datos reportados en las estadísticas continuas del formato 911, menciona que para el ciclo 2016-2017, $56.5 \%$ de las escuelas primarias y $57.9 \%$ de las escuelas secundarias del país contaban con acceso a internet. Sin embargo, las brechas marcan desigualdades en oportunidades educativas entre estos niveles educativos: $59.2 \%$ de las primarias generales tenían conectividad, mientras que únicamente $25.6 \%$ de las primarias indígenas contaban con conexión a internet. En el nivel de secundaria, 79.4\% de las generales, 70.9\% de las técnicas, 36.8\% de las telesecundarias y 86.9\% de las secundarias para trabajadores estaban conectadas a este recurso tecnológico (INEE, 2019).

Los indicadores de infraestructura tic en las escuelas y de conectividad a internet reflejan un gran rezago si los comparamos con datos internacionales como el Índice de desarrollo de las Tıc, elaborado por la Unión Internacional de Telecomunicaciones (UIT), y compuesto por once indicadores que miden el acceso, uso y las aptitudes de la población de un país hacia las Tic. México, en el 2018, con un porcentaje de 34\%, está por debajo de la media internacional, además de naciones como Colombia y Chile, que cuentan con 40\% y 53\%, respectivamente (ITU, 2018).

Aun si todas las escuelas tuvieran acceso a internet, los desafíos en educación continuarían, pues para que las Tic sean efectivas se necesitan, además de acceso y disponibilidad, modelos pedagógicos óptimos y profesores capacitados. Como menciona Díaz (2013), la incorporación de las Tic no se limita al problema de contar con las herramientas que conforman estas tecnologías (equipos y programas de cómputo), sino a su importancia en construir un empleo propositivo y sistemático de las mismas. Además, Díaz (2014) comenta que las computadoras y herramientas tecnológicas no van a producir innovaciones solas, requieren estar asociadas con métodos educativos novedosos y para ello se requieren cambios en las mentalidades y prácticas socioculturales de los actores principales.

\section{Potencialidad de las tic y la educación en ciencias}

Desde su aparición en el ámbito educativo, las tic han producido grandes expectativas y, como ya se ha mencionó, se han documentado numerosas iniciativas para disminuir la brecha tecnológica y formativa de la educación básica en México. El problema recae en la diferencia entre las elevadas expectativas de trasformación puestas sobre estas herramientas y los limitados logros educativos de los que se tiene evidencia hasta el momento. Sin embargo, varios autores sostienen que las tıc poseen importantes cualidades. Por ejemplo, Coll (2008) sostiene: 
Lo que sucede es que la capacidad de transformación y mejora de la educación de las tic debe entenderse más bien como un potencial que puede o no hacerse realidad, en función del contexto en el que son utilizadas (p. 1).

Al parecer, son los marcos de empleo los que definen el éxito o fracaso de estos medios en la práctica educativa. A continuación, argumentaremos sobre el potencial de las TIC en la educación desde dos perspectivas: la primera, su capacidad para transformar los escenarios educativos actuales, fundamentalmente los llamados de la educación formal, y, la segunda, desde sus facultades para generar la aparición de nuevos modelos educativos.

En el primer caso, consideramos que el enfoque óptimo para mostrar las TIC como un instrumento educativo revolucionario es el sociocultural de la psicología educativa de Vygotski. A partir de dichas propuestas, Coll (2004) suscribe el uso de las TIC, pues afirma que "el aprendizaje sobre todo el intencional que es el que tiene lugar en las situaciones educativas formales, es el resultado de complejos procesos interactivos y comunicativos" (p. 8). También recalca la importancia de los instrumentos utilizados para comunicar y representar la información, con los cuales el aprendiz puede interiorizar, presentar y contrarrestar los conocimientos adquiridos con otros. Esto sin duda muestra las características y propiedades del entorno simbólico o semiótico que las tic ponen a disposición del aprendiz. Por su parte, Da Cruz (2009) considera el desarrollo de la inteligencia como una construcción continua de estructuras y propone que:

[...] la función cognitiva del sujeto lleva a dos tipos de construcciones: Ios sistemas de significaciones y los sistemas lógicos. Los significados son estructurados gradualmente por el razonamiento lógico, que genera conceptos y sistemas de conceptos en el uso de las funciones de adaptación y de organización, invariantes de la vida mental (p. 135).

Todas estas construcciones cognitivas requieren de condiciones apropiadas, tales como actuar e interactuar socialmente con otras personas. Por estas razones las TIC —usadas como objetos para que el aprendiz explore, investigue, cuestione, simule, pregunte, valide, demuestre y formalice-potencializan la capacidad de asimilar la información.

En cuanto a la capacidad de las tıc para facilitar la aparición de nuevos escenarios educativos, es necesario retomar el concepto de sociedad del conocimiento. La unEsco (2005) lo define como "aquella sociedad que tiene la capacidad para identificar, producir, tratar, transformar, difundir y utilizar la información con vistas a crear y aplicar los conocimientos necesarios para el desarrollo humano" (p. 9). En este concepto la relevancia del aprendizaje a lo largo de la vida y la necesidad de nuevas competencias formativas son imprescindibles. Aquí las TIC pueden adquirir un papel fundamental porque traspasan algunas barreras espaciales y temporales, haciendo posible el aprendizaje en escenarios diversos. 
En este sentido, Martín y Marchesi (2006) hablan de la potencialidad de las Tic para la "autonomía de gestión de conocimientos" y la "co-construcción de los aprendizajes". Los autores mencionan que entre los objetivos de la educación está lograr la competencia de "aprender a aprender" en los estudiantes. Si bien resultaría excesivo decir que con la sola presencia de las TIC producirían esta competencia, sí favorecen su aprehensión.

Por último, es necesario enfatizar que las tic son de utilidad a una gran diversidad de individuos. Debido a esto, han sido vitales para apoyar a grupos específicos de estudiantes con necesidades educativas especiales, cuyas diferencias son, de hecho, causa de la desigualdad en el sistema educativo.

En resumen, la capacidad mediadora de las TIC como instrumentos psicológicos y su ubicuidad originan las potencialidades que pueden maximizar los aprendizajes, en medida de las relaciones que se establezcan entre los tres elementos que conforman lo que Coll (2004) Ilamó triángulo didáctico: el contenido, que es objeto de enseñanza y aprendizaje, la actividad educativa e instruccional del profesor y las actividades de aprendizaje de los estudiantes.

\section{Aportaciones de las tıc para la enseñanza en ciencias}

La enseñanza de las ciencias es un proceso complejo a partir del cual se han desarrollado diversos enfoques didácticos. Éstos, a pesar de ser distintos, coinciden en que para enseñar ciencias hay que desarrollar a los estudiantes en dimensiones conceptuales, procedimentales y actitudinales, como menciona Veglia. Por esta causa es necesario poseer:

1. Un cuerpo conceptual de conocimientos; donde se hablan de conceptos, teorías, hechos, entre otros.

2. Un modo de producción del conocimiento; donde se enmarca la observación, experimentación, investigación, formulación de hipótesis, elaboración de conclusiones, etcétera.

3. Un módulo de vínculo con el saber; en el cual se habla de las actitudes de quien investiga: flexibilidad, rigurosidad, manejo de datos (Veglia, 2007, pp. 17-18).

Al enseñar ciencias no deben olvidarse estas tres dimensiones y con base en ellas se propone la utilización de las tic para potencializarlas (ver figura 1). Asimismo, se sugiere, a partir de las propiedades de los entornos simbólicos señaladas por Coll (2004), que su contribución en la enseñanza de las ciencias sea, a su vez, en tres dimensiones:

En primer lugar, para la dimensión conceptual de la educación de las ciencias, se propone la explotación sistemática de dos propiedades de las TIc: la multimedia y la hipermedia. La multimedia permite la integración, complementariedad y el tránsito de información en diferentes formas. La 
"¿Qué aportan las Tecnologías de la Información y Comunicación en la enseñanza de las ciencias?" Héctor Medina Cruz, Agustín Lagunes Domínguez y María Teresa Guerra Ramos

Vol. 21, Núm. 3, mayo-junio 2020 Revista Digital Universitaria

hipermedia, en cambio, brinda la posibilidad de establecer formas múltiples y flexibles de organizar la información, también se apoya de sus características para explotar el conocimiento de maneras independientes y creativas.

Por otra parte, para la dimensión procedimental, el dinamismo que proporcionan las TIc permite trabajar con herramientas que pueden obtener y trabajar con datos y recursos para generar resultados o hallazgos. Sobresale, de igual modo, la opción de emplear simulaciones de situaciones reales, favoreciendo así la exploración y la experimentación de los estudiantes al relacionarse con temas científicos.

Finalmente, se explicarán las tres propiedades de las tıc capaces de mejorar la integración de contenidos actitudinales en la enseñanza de las ciencias. Una de ellas es el formalismo, que implica la planificación de las actividades favoreciendo la toma de conciencia y la autorregulación. La conectividad da pie al trabajo en red por lo que abre la posibilidad de trabajo grupal y colaborativo en cualquier parte, facilitando la diversificación en la cantidad y calidad de enseñanza. Por último, la interactividad facilita que los estudiantes tengan una relación más activa con la información, lo cual permite distintos ritmos de aprendizaje, beneficiando la motivación y la autoestima.

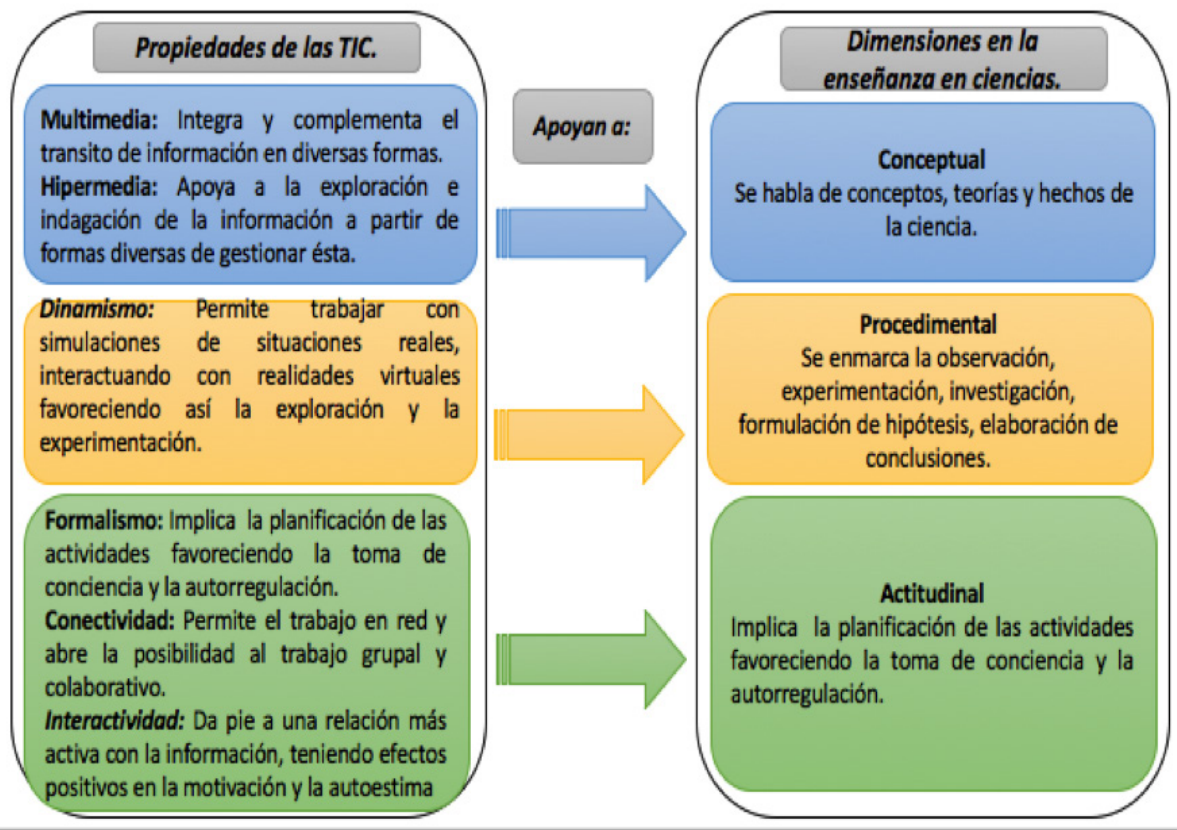

No obstante, los escenarios educativos que saquen partido de estas propiedades sólo son posibles con la integración de las tıc en la enseñanza de las ciencias en el marco de una aproximación sociocultural clara y bien planificada. Evidentemente, las consideraciones sobre las TIC y su potencial para fortalecer la pedagogía en esta área todavía requieren ser concretadas en propuestas didácticas, las cuales prueben sus bondades en las aulas, donde existen otros factores que inciden en tales procesos. 
"¿Qué aportan las Tecnologías de la Información y Comunicación en la enseñanza de las ciencias?" Héctor Medina Cruz, Agustín Lagunes Domínguez y María Teresa Guerra Ramos

\section{Conclusiones}

La presencia de las tic en los espacios educativos, aunque cambiante e inequitativa, resulta innegable, como lo sugieren los datos presentados. Al menos con lo que respecta a México, un número importante de programas gubernamentales han implementado herramientas tecnológicas en los procesos educativos. Sin embargo, varios autores han señalado que la mera inversión en infraestructura tecnológica no garantiza mejores resultados, pues hay otras circunstancias a considerar como los usos que le dan los profesores y la necesidad de una perspectiva pedagógica.

Se han revisado algunas características de las tic que las posicionan como instrumentos mediadores entre los contenidos educativos y el aprendizaje de los estudiantes para lograr que éstos adquieran mejor los conocimientos. También, se ha destacado el enfoque socioconstructivista de la enseñanza en ciencias para lograr estudiantes que, a través de la utilización de las tıc, tengan una formación más sólida, la cual les permita tomar decisiones en la vida cotidiana y convertirse en ciudadanos más informados y formados.

Por lo tanto, las propiedades de las TIC con potencial para fortalecer los procesos educativos en ciencias deben estar soportadas en modelos pedagógicos creados para ambientes educativos concretos. Coll (2004) define como diseños tecno-pedagógicos a aquellos en donde los usos pedagógicos efectivos que se hacen a partir de las tic parten tanto de los recursos tecnológicos disponibles y adecuados (diseños tecnológicos) como de la utilización de estos recursos a través del desarrollo de actividades adecuadas de enseñanza y aprendizaje (diseño pedagógico). Esto es coherente con los planteamientos más vanguardistas de la didáctica de las ciencias y la perspectiva sociocultural, los cuales estipulan que el aprendizaje profundo y relevante de las ciencias únicamente podrá ocurrir en ambientes que privilegien la interacción significativa entre los participantes, en torno a un tema con trascendencia social. Así, se favorece la socialización de los conocimientos en el plano interpersonal y en el intrapersonal.

Entonces, podemos aprovechar las características de las tic para apoyar estos procesos y superar el dominio de la información como fin, para avanzar hacia la comprensión, la conciencia y la acción. La enseñanza de las ciencias, con o sin Tıc, debe aspirar a la formación integral de los estudiantes, prepararlos para ser activos en una sociedad versátil en donde que la ciencia forma parte.

Nuestra intención es alimentar la discusión en este tema y plantear las bases con el objetivo de progresar en el desarrollo de diseños tecno-educativos coherentes y útiles para profesores y estudiantes. 
“Qué aportan las Tecnologías de la Información y Comunicación en la enseñanza de las ciencias?" Héctor Medina Cruz, Agustín Lagunes Domínguez y María Teresa Guerra Ramos

Vol. 21, Núm. 3, mayo-junio 2020 Revista Digital Universitaria

\section{Referencias}

* Aikenhead, G. (2003). sts education: A rose by any other name. En G. Aikenhead (Ed.), A vision for science education. Responding to the work of Peter Fensham (pp. 114-124, E. Arjonilla,Trad.). RoutledgeFalmer. http://citeseerx.ist.psu.edu/viewdoc/ downl oad?doi=10.1.1.464.1180\&rep=rep1\&type=pdf.

* Calixto Flores, R., y Rebollar Albarrón, M. A. (2008). La Telesecundaria, ante la sociedad del conocimiento. Revista Iberoamericana de Educación, 44(7), 1-11. http:// rieoei.org/2197.htm.

- Castañeda Castañeda , A., Carrillo Álvarez , J., y Quintero Monreal , Z. Z. (2013). El uso de las tic en Educación Primaria: la Experiencia ENCICLOMEDIA. ReDIE. http:// redie.mx/librosyrevistas/libros/usoticseducprim.pdf.

* Coll, C. (2008). Aprender y enseñar con las tic: expectativas, realidad y potencialidades. Boletín de la Institución Libre de Enseñanza, 72, 113-126. https:// www.educ.ar/recursos/70819/aprender-y-ensenar-con-las-tic-expectativasrealidad-y-potencialidades.

* Coll, C. (enero-agosto de 2004). Psicología de la educación y prácticas educativas mediadas por las tecnologías de la información y la comunicación. Una mirada constructivista. Revista Electrónica Sinéctica, 25, 1-24. http://www.redalyc.org/ pdf/998/99815899016.pdf.

- Da Cruz Fagundes, L. (2009). Las condiciones de la innovación para la incorporación de las tic en la educación. En R. Carneiro, J. C. Toscano, y T. Díaz (Eds.), Los desafíos de las tic para el cambio educativo (pp. 127-138). Fundación Santillana. http:// www.fundacionsantillana.com/upload/ficheros/paginas/200906/xxii_semana $\underline{\text { monografica.pdf }}$

- Díaz Barriga Arceo, F. (2014). Las políticas tic en los sistemas educativos de América Latina, Caso México. unicef-oel.

- Díaz Barriga, Á. (2013). tıc en el trabajo del aula. Impacto en la planeación didáctica. Revista Iberoamericana de Educación Superior, iv(10), 3-21.

http://dx.doi.org/10.1016/S2007-2872(13)71921-8.

* García Cué, J. L., y Santizo Rincón, J. A. (2006). Integración de tic en México. Colegio de Postgraduados. http://www.jgcue.es/ticmex.pdf.

* Instituto Nacional para la Evaluación de la Educación (InEe). (2019). Panorama Educativo de México 2018 Indicadores del Sistema Educativo Nacional Educación Básica y Media superior I [B. Gayosso, Ed.]. INEE. https://www.inee.edu.mx/wpcontent/uploads/2019/08/P1B117.pdf.

* International Telecommunication Union. (2018). Measuring the Information Society Report, I. ITU. https://www.itu.int/en/ITUD/Statistics/Documents/publications/ misr2018/MISR-2018-Vol-1-E.pdf.

- Lugo, M. T., Kelly, V., y Schurmann, S. (2012). Políticas tic en educación en América Latina: más allá del modelo 1:1. Revista Cientí ica de Tecnología Educativa, I (01), 1-12. http://www.uajournals.com/campusvirtuales/journal/1/3.pdf. 
* Martín Ortega, E., y Marchesi Ullastres, Á. (2006). Las tecnologías de la información y la comunicación y los procesos de aprendizaje. Propuestas de introducción en el curriculum de las competencias relacionadas con las TIC. UNESCO. http://unesdoc. unesco.org/images/0015/001507/150785s.pdf.

* Organización de las Naciones Unidas para la Educación, la Ciencia y la Cultura (unesCo). (2005). Hacia las sociedades del conocimiento. unEsco. http://unesdoc. unesco.org/images/0014/001419/141908s.pdf.

* Secretaría de Educación publica (sep). (2016).Programa @prende 2.0. Programa de Inclusión Digital 2016-2017. México. Recuperado el 2 de enero de 2020. https:// www.gob.mx/cms/uploads/attachment/file/162354/NUEVO PROGRAMA PRENDE 2.0.pdf.

* Secretaría de Educación publica (SEP). (2013). Programa Sectorial de Educación 2013-2018. Impresora y Encuadernadora Progreso (IEPSA). http://www.sep.gob. mx/work/models/sep1/Resource/4479/4/images/PROGRAMA SECTORIAL DE EDUCACION 20132018 WEB.pdf.

* Veglia, S. M. (2007). Didáctica de las ciencias naturales: Una reflexión crítica del área. En Ciencias naturales y aprendizaje significativo: claves para la reflexión didáctiva y la planificación (pp. 13-48). Novedades Educativas.

\section{Cómo citar el artículo}

* Medina Cruz, Héctor, Lagunes Domínguez, Agustín y Guerra Ramos, María T. (2020). ¿Qué aportan las Tecnologías de la Información y Comunicación en la enseñanza de las ciencias? Revista Digital Universitaria (RDU) Vol. 21, núm. 3 mayojunio. Dol: http://doi.org/10.22201/codeic.16076079e.2020.v21n3.a9.

Recepción: 16/08/2018. Aceptación: 12/11/2019. 